\title{
Interactive comment on "Wuhan MST radar: Technical features and Validation of wind observations” by Lei Qiao et al.
}

\section{Lei Qiao et al.}

qiaolei@hdu.edu.cn

Received and published: 1 May 2020

General Comments:

Answer: Dear reviewer, this paper mainly focus on the system description of the Wuhan MST radar. Because Chen et al. (2016) has introduce the antenna array of the Wuhan MST radar, we mainly introduce the technical features in this paper. It includes antenna field, timing signal, TR module, digital transceiver and clutter suppression. Then we briefly analyze some cases and long tern comparisons. This part is the preliminary work of validation. We would like to thank the reviewer for valuable and constructive comments and suggestions. We have revised the paper in line with the reviewer's comments, thereby improving the technical quality and the clarity of the paper accordingly. 
Specific Comments:

(1)The MST radars from China were discussed at length in few system related papers AMTD (Chen et al. 2016). What is new in this paper? Is there any upgrade made after those papers? If the authors intention is to highlight the stable performance of the system, then it is better to do a detailed scientific evaluation.

Answer: Dear reviewer, the RF circuits of TR modules were optimized, and the detailed description is shown in the paper. Meanwhile, the inter connections of the shelter and the feeding network were modified. Monitoring information of the small TR modules are shown in Figure 1. The red square represents the damaged TR module, and the green square represents the good TR module. It is obvious that the damaged TR modules decrease significantly after the upgrade. Therefore, the Wuhan MST radar is in good running condition after the upgrade.

(2)Lines 30-37: Several of these radars have been upgraded, like MU radar, Indian MST radar, NERC MST radar, etc. It is better to include recent references also to have updated knowledge on these radars.

Answer: Thank you for your suggestion. We updated the references in the revised paper.

(3)The description of the system is not complete. Enough details were not provided on the antenna parameters, TR module specifications and RF performance. Also, it is better to include important specifications of the system in a table.

Answer: Thank you for your suggestion. We listed the important specifications of the system in table 1 in the revised paper, including the antenna parameters, TR module specifications, RF performance and so on.

(4)A separate sub-section exists on clutter suppression without describing how it is done! Is it simple removal of data at zero frequency and fill it with interpolated data from neighboring points? Or do you employ any filtering techniques (like wavelets)?

Interactive

comment

Printer-friendly version

Discussion paper 
Answer: Dear reviewer, the wavelet method is usually used in time-domain, and the ground clutter suppression used here is based on frequency-domain. The effectiveAMTD ness of the algorithm is satisfactory.

(5)In spite of having two years of observations, the authors restricted the analysis to one profile comparison. Even that comparison shows a difference of 5-7 m s-1 in the mid- and upper-troposphere, too large to accept. The authors should do the validation using a large data set to have a statistically robust conclusion on the performance of the radar.

Answer: Dear reviewer, the radiosondes were launched by us on 22 May 2016, which are not from the standard observatory. Therefore, we don't have a large data set of the radiosondes, and it is difficult to do a long-term comparison between the Wuhan MST radar and the radiosonde. That's why we compare the mean zonal and meridional winds from the Wuhan MST radar and the ERA-interim, and the results are in good agreement at heights of $3.5-25 \mathrm{~km}$. The difference of the results between the Wuhan MST radar and the radiosonde is due to the different measurement principles. The radiosonde will not just pass the detection area of the MST radar, and there is a difference of over 100 kilometers. So the comparison shows a difference of several meters, which is normal.

(6)Line 289: Several reasons were quoted for the wind discrepancy, including aspect sensitivity, without dwelling on any of those issues. Mere quoting of some references (elsewhere) may not resolve the problems in your radar or analysis. If aspect sensitivity is the real reason, why is it occurring only at those heights and in meridional plane alone?

Answer: Dear reviewer, the radiosondes were launched by us on 22 May 2016, which are not from the standard observatory. Therefore, there is only one profile comparison. Therefore, we can only introduce many possible reasons. The concrete reason needs more profile comparisons to analyze, which is our next work. I hope to get your

Interactive comment 
understanding.

(7)Line 308: Even the average wind difference between the radar and ERA is too large AMTD (10 ms-1). What could be the reason for this difference? Also, do some statistical analysis by providing RMSE and correlations with statistical significance tests.

Answer: The EAR radar is at the Indonesian equator $\left(10.63^{\circ} \mathrm{S}\right)$, and the Wuhan MST radar is at latitude $29.5^{\circ} \mathrm{N}$. The difference from the EAR radar observation is probably that the two radars are at different latitudes and in different atmospheric circulation. Considering the latitude difference, it is difficult to do the correlation analysis of the two radars. I hope to get your understanding.

(8)Line 354: Same problem as above, the SSW events were cited as the potential reason for the wind discrepancy without verification. Instead of citing old references, why don't you check whether or not any such events occurred during that period?

Answer: Thank you for your suggestion. Fig. 2(a) shows the time-altitude evolution of the daily mean zonal wind observed by the Wuhan MST radar from 66 to $86 \mathrm{~km}$ during 2016 SSW winter (Jan to Feb). The 2016 Feb SSW is a minor SSW, and the day of peak warming on Feb 5 is marked by the dotted vertical line. The wind weakening is observed around Feb 5 . Note that the westward wind form 68 to $78 \mathrm{~km}$ during Jan 10 to Jan 14 is a reversal of the climatological mean zonal wind, which has nothing to do with the SSW. Fig. 2(b) shows the time-altitude evolution of the daily mean zonal wind observed by the Wuhan MST radar from 66 to $86 \mathrm{~km}$ during 2017 SSW winter (Jan to Feb). Two minor warming events happened during the winter of 2017, with two days of peak warming on Feb 2 and 26, marked by dotted vertical lines in the figure. The wind reversal is observed around Feb 2, and the wind weakening is observed around Feb 26 (not obvious). This is a preliminary analysis. Considering the discussion of SSW is not the gist of the paper, the figure will be used as a supplementary material.

(9) So many grammatical errors to list here (few of them are given below in minor comments). They should be corrected before the submission of the revised version.

Interactive

comment

Printer-friendly version

Discussion paper

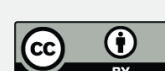


Answer: Dear reviewer, we modified grammatical errors in the revised paper.

Minor Comments:

AMTD

(1)Lines 13-14: Rewrite these sentences.

Answer: Thank you for your suggestion. We rewrite the sentences in the revised paper.

(2)Line 26: Change to "The mesosphere-stratosphere-troposphere (MST) radars have been used for studying the......"

Answer: Thank you for your suggestion. We modified it in the revised paper.

(3)Line 29: Replace 'applied' with 'employed' or some other suitable word. Same line, should be 'turbulence'

Answer: Thank you for your suggestion. We modified them in the revised paper.

(4)Line 31: The sentence is abruptly ending. MST community plays a significant role in what?

Answer: Thank you for your suggestion. We modified the sentence in the revised paper.

(5)Lines 38-39: Rewrite these sentences.

Answer: Thank you for your suggestion. We rewrite the sentences in the revised paper.

(6)Line 44: Should be '....to write a new article in response to the readers and users demand (or request)...'

Answer: Thank you for your suggestion. We modified the sentence in the revised paper.

Printer-friendly version

(7)Line 49: Remove 'of radar echoes'

Answer: Thank you for your suggestion. We modified the sentence in the revised paper. 
(8)Line 74: The signal is scattered by 'refractive index irregularities'.

Answer: Thank you for your suggestion. We modified the sentence in the revised AMTD paper.

(9)Line 99: With $4 \mathrm{~m}$ antenna spacing, one can tilt the beam up to $24^{\circ}$ from zenith without grating lobe!!.

Interactive

comment

Answer: Thank you for your suggestion. We modified the angle in the revised paper.

(10)Line 114: 'respects' is not the correct word there.

Answer: Thank you for your suggestion. We modified the word in the revised paper.

(11)Line 115: ...data pots of .... Correct it.

Answer: Thank you for your suggestion. We modified it in the revised paper.

(12)Line 154: How about azimuth angles?

Answer: Dear reviewer, the oblique beams point to the north, south, west and east, and the azimuth angles corresponds to $90^{\circ}, 180^{\circ}, 270^{\circ}, 360^{\circ}$.

(13)Line 174: The recovery time of $T / R$ switch is somewhat on higher side, which restricts the minimum height coverage (if shorter pulses are available)

Answer: The minimum detecting height of the low mode is $3.5 \mathrm{~km}$, and the propagation time $(23 \mu \mathrm{s})$ is greater than the recovery time of $\mathrm{T} / \mathrm{R}$ switch.

(14)Line 218: Replace 'in sunny day' with 'during fair weather'

Answer: Thank you for your suggestion. We modified it in the revised paper.

(15)Line 210: Since the LNA bandwidth of small TR module is $1 \mathrm{MHz}$, FIR filter bandwidth of $1.5 \mathrm{MHz}$ will not improve the performance. First of all, what is the logic in choosing $1 \mathrm{MHz}$ bandwidth at LNA?

Printer-friendly version

Answer: Dear reviewer, the shortest pulse is $1 \mu \mathrm{s}$, so the LNA bandwidth is $1 \mathrm{MHz}$. We 
made a mistake, and the FIR filter bandwidth is $1 \mathrm{MHz}$. We modified it in the revised paper.

(16)Line225: Should be 'Doppler spectra'. The sentences in this paragraph suffer with several grammatical errors. Correct them.

Answer: Thank you for your suggestion. We modified them in the revised paper.

Interactive

comment

(17)Line 230: What do you mean by high-frequency interference?

Answer: Dear reviewer, the high frequency interferences refer to internal noise of the radar.

(18) Line 231: Bring more clarity in presentation. At present, description of different modes of operation exists under 'Validation of wind observations'. Add one more subsection 3.1. Modes of operation and then change numbers of other subsections accordingly.

Answer: Thank you for your suggestion. We modified them in the revised paper.

(19)Line 245: If the temporal resolution of the data is $30 \mathrm{~min}$, then the number of data points in a day should be 48 . Then how come different numbers for different modes?

Answer: Dear reviewer, the Wuhan MST radar is down for maintenance periodically, and the radar system sometimes runs in the high mode. Therefore, there are different numbers for different modes.

(20)Line 280: The radiosonde generally take an hour to reach $18 \mathrm{~km}$ assuming an ascent rate of $5 \mathrm{~m} \mathrm{~s}-1$. Is it a special sonde (or filled with more gas?) that reaches 25 $\mathrm{km}$ in 1 hour?

Answer: Dear reviewer, the radiosonde is the normal digital radiosonde filled with more gas.

Discussion paper

(21)Line 300: Which one is latest? ERA-Interim or ERA5? 
Answer: Dear reviewer, EAR-5 is latest, and we modified it in the revised paper.

(22)Line 334-336: Rewrite the sentences. Also the data acquisition rate is high at 75 AMTD $\mathrm{km}$ not at $80 \mathrm{~km}$.

Answer: Thank you for your suggestion. We modified the sentences in the revised paper.

Interactive comment

(23)Line 351-354: Rewrite the sentences.

Answer: Thank you for your suggestion. We modified the sentences in the revised paper.

Please also note the supplement to this comment: https://www.atmos-meas-tech-discuss.net/amt-2020-17/amt-2020-17-AC2supplement.pdf

Interactive comment on Atmos. Meas. Tech. Discuss., doi:10.5194/amt-2020-17, 2020. 


\section{AMTD}

Interactive comment

Before the upgrade

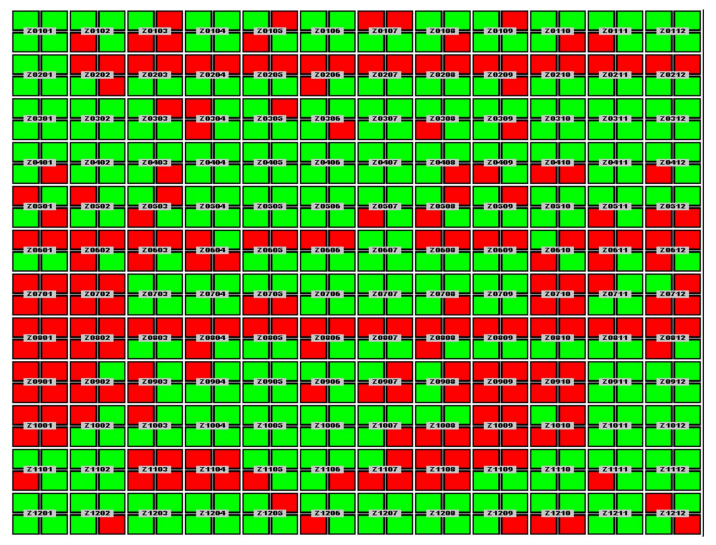

(a)
Six months after the upgrade

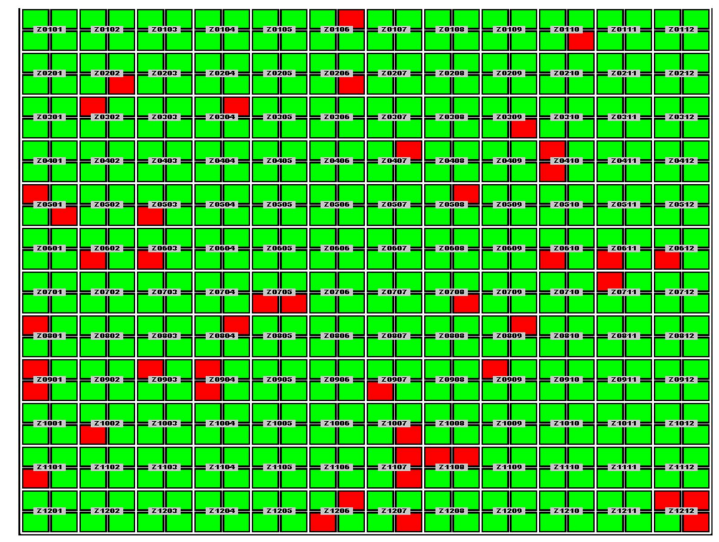

(b)

Fig. 1. Monitoring information of the small TR modules. 
AMTD

(a)

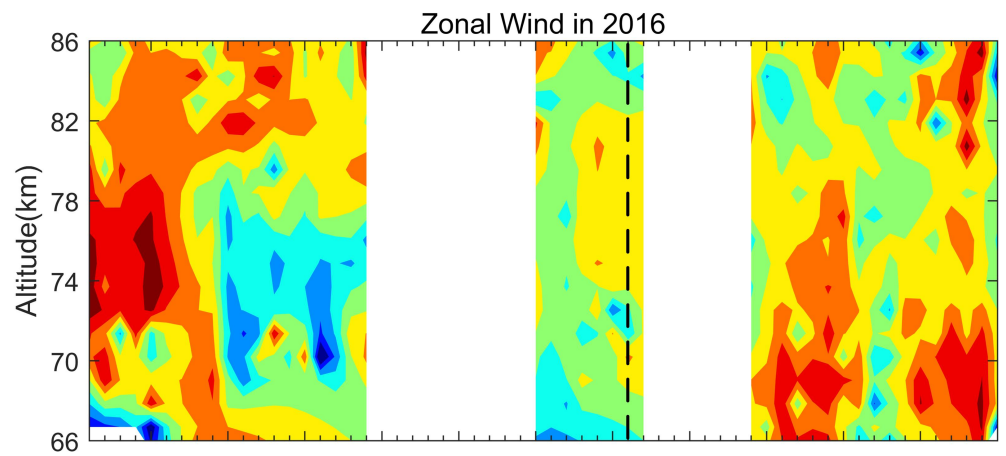

Interactive comment

(b)

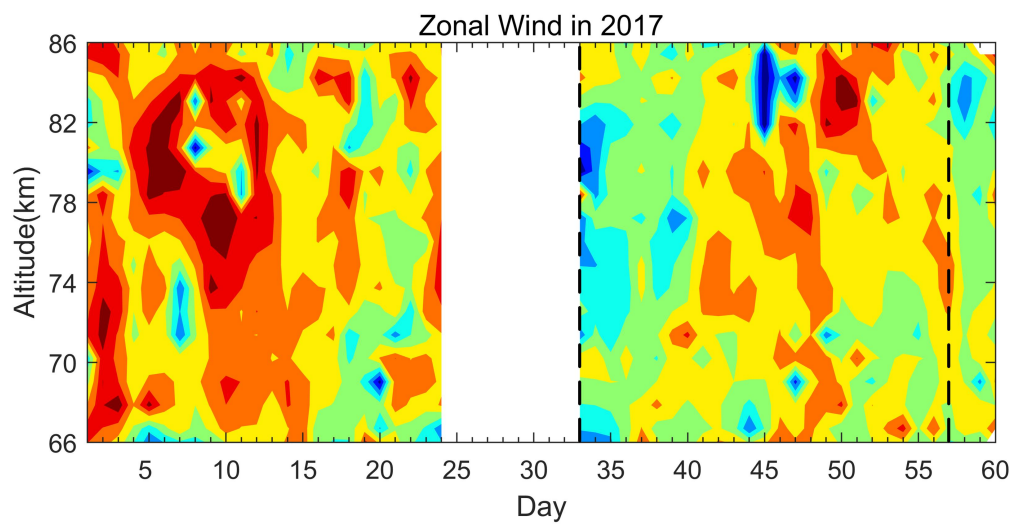

$-50$

50

Fig. 2. Time-altitude evolution of the daily mean zonal winds observed by the Wuhan MST radar from 66 to $86 \mathrm{~km}$ during Jan to Feb in 2016 (a) and 2017 (b). The dotted vertical lines indicate peak warming. 